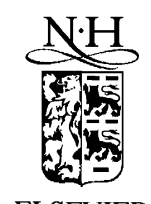

Available online at www.sciencedirect.com

ELSEVIER

Journal of Magnetism and Magnetic Materials 294 (2005) e99-e103

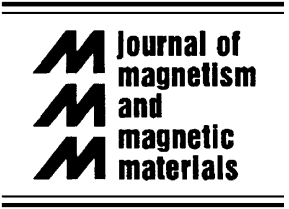

www.elsevier.com/locate/jmmm

\title{
Evolution of magnetoresistance effect in iron-bismuth films
}

\author{
Jen-Hwa Hsu ${ }^{\mathrm{a}, *}$, Hong-Xian Wang ${ }^{\mathrm{b}}$, P.C. Kuo ${ }^{\mathrm{b}}$ \\ ${ }^{a}$ Department of Physics, National Taiwan University, Taipei 106, Taiwan \\ ${ }^{\mathrm{b}}$ Department of Material Science \& Engineering, National Taiwan University, Taipei 106, Taiwan
}

Available online 21 April 2005

\begin{abstract}
A unique time-dependent magnetotransport behavior has been observed in $\mathrm{Fe}-\mathrm{Bi}$ films. Approximately one month was required to stabilize resistance after samples were fabricated. Initially, the as-prepared $\mathrm{Fe}-\mathrm{Bi}$ films exhibit both ordinary magnetoresistance (MR) and anisotropic magnetoresistance. Fe precipitation changes the microstructure. Eventually, an isotropic and negative MR, like a giant MR effect was observed; it arises from electronic transport between $\mathrm{Fe}$ granules separated by Bi.
\end{abstract}

(C) 2005 Elsevier B.V. All rights reserved.

PACS: 75.47.-m.; 81.30.Mh.; 81.05.Bx.; 81.40.Cd

Keywords: Magnetotransport; Precipitation; Semimetal; Aging

\section{Introduction}

$\mathrm{Bi}$ is almost immiscible with Fe. However, the metastable $\mathrm{Fe}-\mathrm{Bi}$ solid solution can be prepared by rapid quenching [1]. Fe is a typical ferromagnetic material and $\mathrm{Bi}$ is a diamagnetic metal, so mixtures of these two metals with diametrically different magnetic characteristics give raise to interesting and peculiar magnetic and transport properties [2]. Furthermore, our previous investigations have revealed that as-made $\mathrm{Fe}-\mathrm{Bi}$ alloy

\footnotetext{
*Corresponding author. Tel.: + 886233665162 ; fax: +886233665892 .

E-mail address: jhhsu@phys.ntu.edu.tw (J.-H. Hsu).
}

film exhibits numerous time-dependent magnetic behaviors [3]. For instance, the as-made sample exhibits large out-of-plane anisotropy, which disappears after a long period of aging. The instability of these as-prepared films is caused by the precipitation of $\mathrm{Fe}$ atoms during natural aging. The variation of saturation magnetization and coercivity depends on the concentration of Fe.

This study continues to examine the magnetotransport behavior of $\mathrm{Fe}-\mathrm{Bi}$ composite films. Precipitation changes the magnetoresistance (MR) effect. Before the films have reached their final equilibrium phase, the measured MR effect changes with time and eventually becomes dominated by a negative isotropic MR, like giant magnetoresistance (GMR). 


\section{Experimental}

Metastable $\mathrm{Fe}_{x} \mathrm{Bi}_{1-x}$ alloy films $(0.3 \leqslant x \leqslant 0.5)$ were prepared by DC sputtering using composite targets. The base vacuum was better than $1 \times 10^{-6}$ Torr. The difference between the sputtering rates of $\mathrm{Fe}$ and $\mathrm{Bi}$ is quite large, so presputtering was performed for a long time before the formal deposition procedure was started. $\operatorname{Si}\left(\begin{array}{lll}1 & 0 & 0)\end{array}\right)$ wafers were used as the substrate. The thickness of the film was around $1 \mu \mathrm{m}$. In this work, $x$ denotes the relative volume fraction of Fe.

Crystalline structures were determined by X-ray diffraction (XRD). $\mathrm{CuK}_{\alpha}$ radiation was used. Microstructures were determined by transmission electron microscopy (TEM). An electron probe $\mathrm{X}$-ray micro-analyzer (EPMA) was used to ascertain the composition of the films. A conventional four-probe method was used to determine resistivity between 10 and $300 \mathrm{~K}$. The MR ratio, $[\rho(H)-\rho(0)] / \rho(0)$, was measured at room temperature with a maximum applied field of $1 \mathrm{~T}$.

\section{Results and discussion}

As stated in Ref. [3], films of as-made $\mathrm{Fe}_{x} \mathrm{Bi}_{1-x}$ contain a single rhombohedral phase that is mostly bismuth. Aging for a long time does not noticeably change the structure, even though $\mathrm{Fe}$ nanograins are randomly distributed in the Bi matrix, because the scattering factor of $\mathrm{Bi}$ greatly exceeds that of Fe. During natural aging, Fe nanograins begin to precipitate out; their size, as determined from TEM images, is less than $3 \mathrm{~nm}$. A dead surface layer forms on the surface of $\mathrm{Fe}$ nanograins and reduces the magnetization of the films.

Fig. 1 plots the dependence of film resistivity on aging time. Initially, resistivity increasing steadily with aging time to a stable value that is reached after around one month; the percentage change in resistance is larger for higher $\mathrm{Fe}$ concentration. Phase segregation typically reduces the overall resistance of the sample. In this case, an opposite result was observed; such a result, however, is not unusual. We suggest that the interface of the precipitated $\mathrm{Fe}$ grains in $\mathrm{Bi}$ is very highly resistive and as a result after the formation nanograins

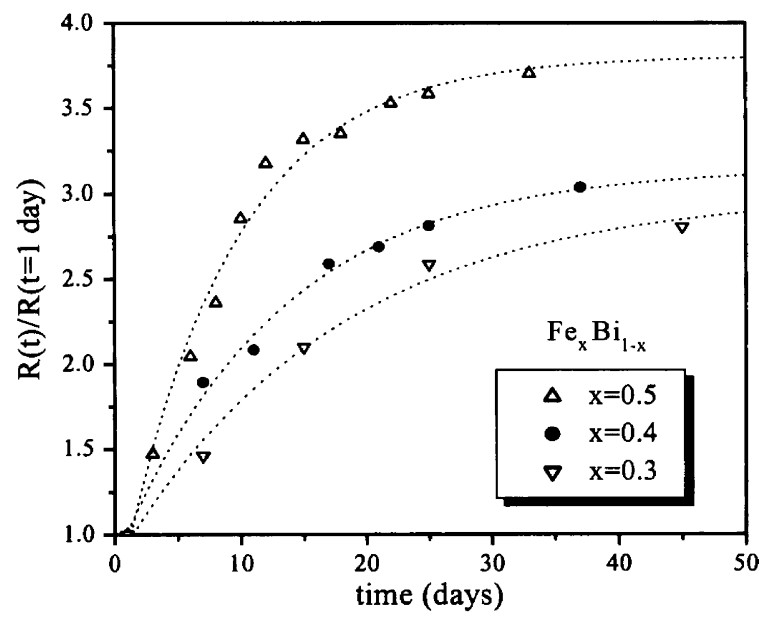

Fig. 1. Variation of resistance at room temperature as a function of aging time, for the samples with various $\mathrm{Fe}$ concentrations.

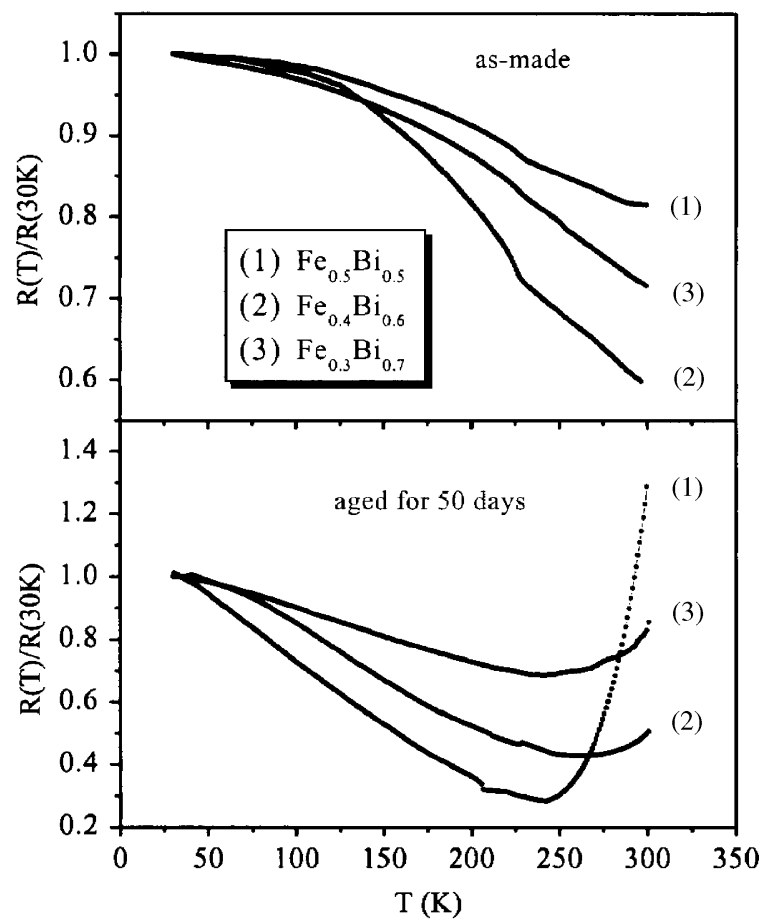

Fig. 2. Resistance as a function of temperature for as-made and aged samples.

electrical current flows mostly through the $\mathrm{Bi}$ matrix. The possibility of oxidation has been excluded. The time-dependence of resistance can 

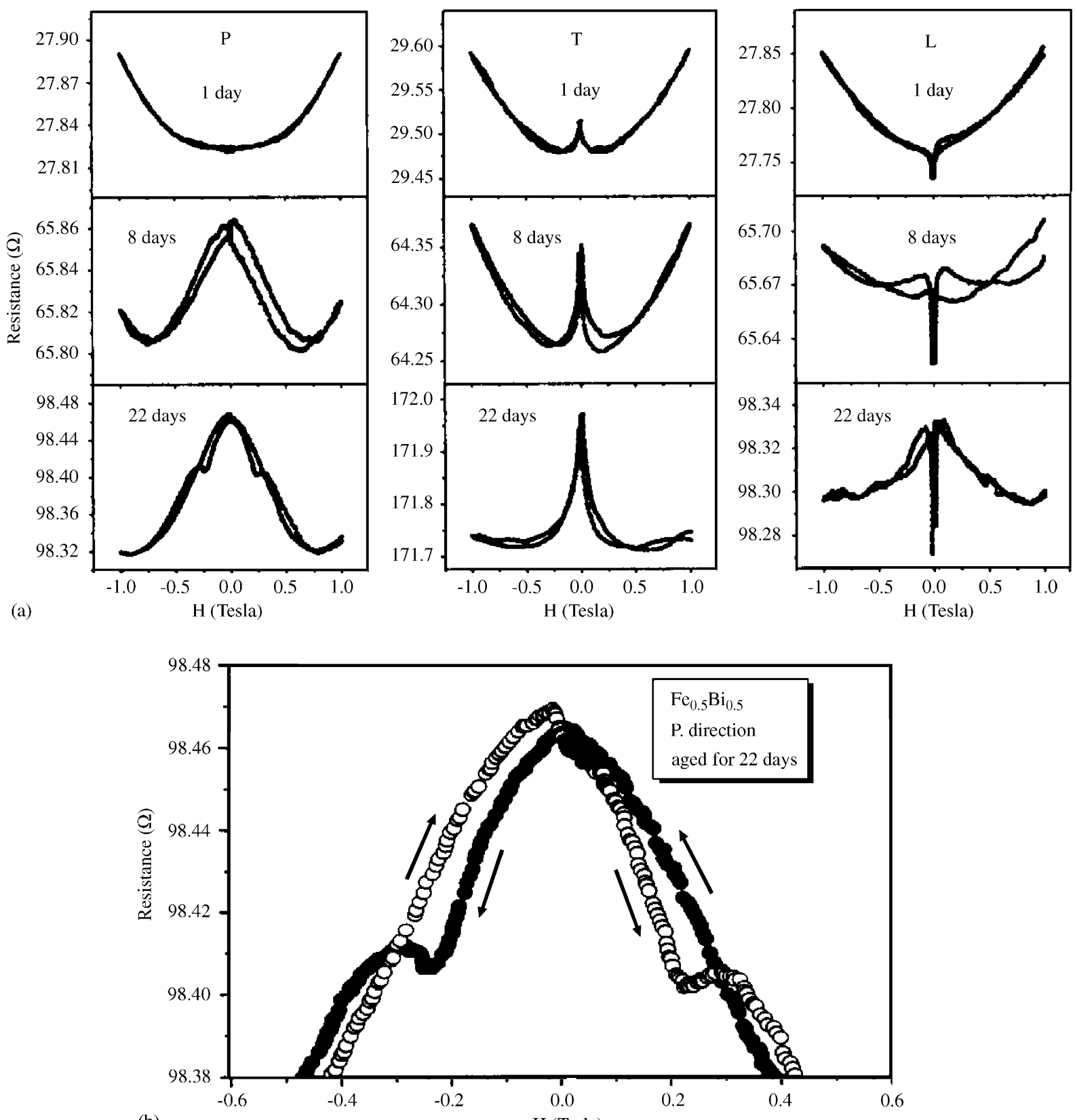

(b)

$\mathrm{H}$ (Tesla)

Fig. 3. (a) MR curves of $\mathrm{Fe}_{0.5} \mathrm{Bi}_{0.5}$ at room temperature in the perpendicular, transverse and longitudinal directions at various stages of aging; and (b) the inner peak of the MR curve in the perpendicular direction for $\mathrm{Fe}_{0.5} \mathrm{Bi}_{0.5}$ aged for 22 days.

be fitted by the equation

$R(t)=R_{0}-C \exp (-\alpha)$ with $\alpha=0.114,0.078$, and 0.058 for $x=0.5,0.4$, and 0.3 , respectively. Fig. 2 plots the temperature dependence of resistivity. The resistance of 
as-prepared samples increases as the temperature declines like that of a semiconductor. After 50 days of aging, a minimum was found for all three samples in the observed temperature range, because of the combination of contributions from metal and semiconductor channels that are in series. The metallic behavior is related to the precipitated atoms so the minimum appears at lower temperatures as the Fe concentration increases.

Fig. 3 plots the magnetoresistance measurements of $\mathrm{Fe}_{x} \mathrm{Bi}_{1-x}$ measured at $300 \mathrm{~K}$, in the perpendicular, transverse and longitudinal directions. For the as-prepared samples, the MR curve displays ordinary magnetoresistance (OMR) with a small anisotropic magnetoresistance (AMR) at low fields. Several days later, a negative MR effect begins to arise and AMR develops to a larger part. Eventually, except at small fields, the resistance decreases as the external field increases in all three measured directions, like a GMR in a granular film [4] but of much smaller magnitude. Compared with the $M-H$ loop of the sample, the resistance of the sample is proportional to $\left(M / M_{\mathrm{s}}\right)^{2}$ at large fields [5]. Therefore, the MR effect originates from spin-dependent scattering between two magnetic $\mathrm{Fe}$ nanograins embedded in the $\mathrm{Bi}$ matrix. A separate report on the magnetotransport between two ferromagnetic layers separated by a semimetallic Bi will be published. As the Fe concentration decreases, a negative MR effect appears at an earlier stage of precipitation process because the precipitation process proceeds more quickly as the Fe concentration declines. Moreover, in this stage, the MR effect of the samples at high fields is still dominated by OMR [6]; the resistance increases with the strength of the field. It is from $\mathrm{Bi}$ matrix. In Fig. 3(b), a dip is observed in the middle of a $R-H$ curve, which appears in both measured directions. This phenomenon can be ascribed to the magnetic dipole interactions between $\mathrm{Fe}$ particles, which decreases the scattering rate and thus decrease the resistance [7].

Fig. 4 plots the MR ratio as a function of aging time for the films with $x=0.5,0.4$, and 0.3 with the field applied in the transverse direction. The MR ratio is defined as $[R(H=1 \mathrm{~T})-R(H=0)] /$ $R(H=0)$. It is noted that the curve starts from a positive value and then it falls. Eventually it

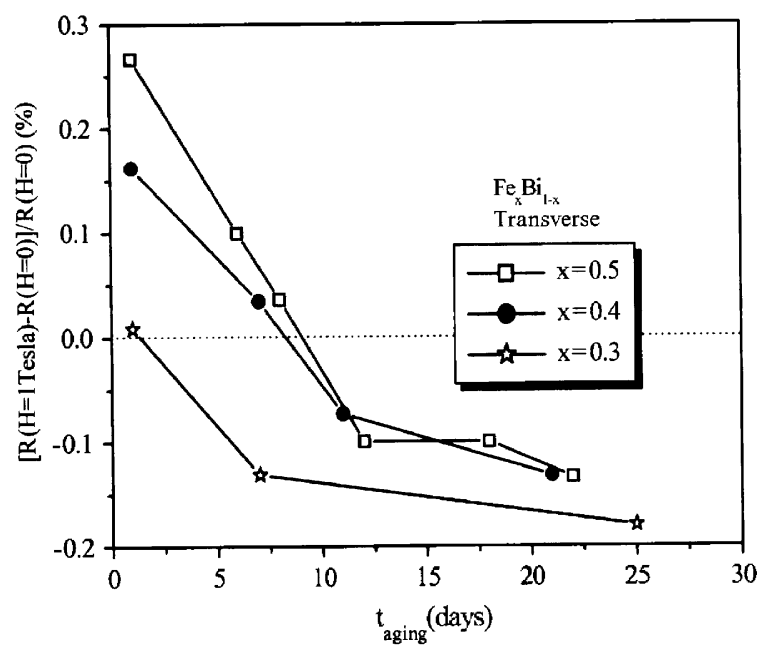

Fig. 4. MR ratio measured in the transverse direction, as a function of aging time for various $\mathrm{Fe}$ concentrations.

becomes negative. The curve crosses over at earlier times for smaller $x$, as discussed above. Additionally, the magnitude of the final MR value is greatest at $x=0.3$ after the OMR has been subtracted. This result agrees with the finding that the granular system with $x \sim 0.3$ has a maximal GMR [8]. The drop of MR for $x>0.3$ is due to the increasing magnetic interactions between Fe particles, which will reduce spin-dependent scattering.

In summary, time-dependent magnetotransport behavior in $\mathrm{Fe}-\mathrm{Bi}$ films is observed. Due to precipitation process, the MR characteristics change with time. OMR is observed initially but the magnetoresistance evolves into a negative and isotropic magnetoresistance, like GMR.

\section{Acknowledgments}

This work was supported by the National Science Council, ROC, under Grant no. NSC922112-M-002-030.

\section{References}

[1] Q.M. Chen, F.Z. Cui, Y.D. Fan, H.D. Li, J. Appl. Phys. 63 (1988) 2452. 
[2] D.W. Forester, J.H. Schelleng, P. Lubitz, P. D’Antonio, C. George, J. Appl. Phys. 53 (1982) 2240; F.Z. Cui, Y.D. Fan, Y. Wang, A.M. Vredenberg, H.J.G. Draaisma, R. Xu, J. Appl. Phys. 68 (1990) 701; S. Honda, Y. Nagata, J. Appl. Phys. 93 (2003) 5538.

[3] Jen-Hwa Hsu, H.X. Wang, A.C. Sun, P.C. Kuo, Appl. Phys. Lett., to be published.

[4] J.Q. Xiao, J.S. Jiang, C.L. Chien, Phys. Rev. Lett. 68 (1992) 3749 ;
A.E. Berkowitz, J.R. Mitchell, M.J. Carey, A.P. Young, S. Zhang, F.E. Spada, F.T. Parker, A. Hutten, G. Thomas, Phys. Rev. Lett. 68 (1992) 3745.

[5] L. Sheng, D.Y. Xing, Mod. Phys. Lett. B 7 (1993) 1365.

[6] J.-H. Hsu, Y.S. Sun, H.X. Wang, P.C. Kuo, T.H. Hsieh, C.T. Liang, J. Magn. Magn. Mater. 272 (2004) 1769.

[7] V. Franco, X. Batlle, A. Labarta, J. Appl. Phys. 85 (1999) 7328.

[8] D. Kechrakos, K.N. Trohidou, Phys. Rev. B 62 (2000) 3941. 